\title{
PAPERS
}

\section{Clinical research: the pharmaceutical industry's point of view}

\author{
F. J. MILLS
}

Senior Medical Adviser, Fanssen Pharmaceutical Ltd

\begin{abstract}
'In research the horizon recedes as we advance, and is no nearer at sixty than it was at twenty. As the power of endurance weakens with age, the urgency of the pursuit grows more intense ... and research is always incomplete.' Mark Pattison (1813-1884).
\end{abstract}

\section{INTRODUCTION}

If research can be likened to a voyage to an unreachable horizon, then in the minds of many research-minded physicians the pharmaceutical industry appears as a pirate ready to reap treasure from this sea of endeavour. In reality such parasitism is not the desire of the industry, for both industry and the medical profession depend intimately upon each other for the development of new and better drugs. Such a state of symbiosis can only be achieved if physicians have some understanding of how the industry views research. This article, based on a lecture given recently to the Emergency Medicine Research Society, aims to provide such a perspective.

\section{Pharmaceutical research}

In 1982, the most recent year for which figures are available, the pharmaceutical industry spent $£ 419$ million on medical research in the United Kingdom. This huge expenditure is made despite, and because of, a high risk of failure. Less than $0 \cdot 1 \%$ of newly synthesized chemicals will reach the clinical pharmacologist, because most are eliminated due to inadequate or inappropriate pharmacological activity, unacceptable animal toxicology or a host of other reasons. Failures in one of the three phases of clinical research (Table 1) carried out prior to an application for a product licence limit further the numbers of new chemical entities that reach the market place.

Such daunting prospects of failure coupled with governmental control of profits and, 
Table 1 The phases of pharmaceutical research

\section{Phase}

I Clinical pharmacology: pharmacokinetics and pharmacodynamics measured in healthy volunteers

II Exploratory therapeutic trials in small numbers of closely monitored patients

III Extensive clinical trials on large numbers of patients

IV Post marketing surveillance-long-term studies of morbidity/mortality, multicentre studies to $\Omega$ monitor usage of the drug

more recently, the removal of National Health Service reimbursement for certain $\frac{\curvearrowright}{\Phi}$ categories of drugs will harden industry's attitudes towards collaboration in research. $\frac{3}{2}$. Value for money will be required increasingly in the future and will be obtained only by $\stackrel{N}{\omega}$ achieving the highest standards of professionalism in research. It should be $\vec{\overrightarrow{ }}$ remembered that the ultimate aim of conducting clinical trials is to change clinical ${ }_{0}^{\omega}$ practice. This change should not occur unless the evidence to support it has been? obtained in well-designed trials conducted by competent investigators.

\section{The selection of investigators}

Investigators are recruited by industry either as a result of a direct approach by the company or because investigators have offered their services to the company. In the former case investigators are likely to have the necessary expertise and track record to reassure the company that they are capable of producing high quality research work. It is, for example, vital in phase II work to collaborate with investigators who are highly experienced in the disease area under question since the results of their work will 2 determine whether the company should or should not enter into very costly phase III $\overrightarrow{\vec{B}}$ development.

Investigators who approach the company should give a clear indication of their interests, the facilities available to them and their motives for wishing to undertake $\bar{\Phi}$ research. Research frequently involves working unsociable hours and making repetitive 3 . measurements so that only the most motivated will succeed. Physicians who are looking 3 for support for a proposed MD thesis will undoubtedly have a greater aspiration to succeed than colleagues who have been asked to undertake work so that a third party $₹$ such as their university will receive financial support. If there is a real possibility of $\bigcirc$ constructive collaboration then a member of the company's research department will $\rightarrow$ visit the investigator. The visitor will be a Medical Adviser or a Clinical Research $\stackrel{\text {. }}{.}$ Associate (CRA) who is responsible for clinical trials in the appropriate therapeutic $\tilde{O}$ area. CRAs are usually graduates and not infrequently have a further degree such as an $\mathrm{PhD}$, often coupled with several years of experience in their chosen speciality. CRAs are N occasionally treated as second-class citizens by investigators-such attitudes are unacceptable to those who realize that a medical qualification does not automaticallyo confer upon the holder an ability to conduct research. 


\section{Initial discussions}

Initial impressions are hard to forget particularly if they are bad. If investigators are hurried because they have not allowed much time for the meeting and appear disorganized then they are unlikely to be suited to the disciplines of research. At this first meeting there must be plenty of time to discuss the drug, its likely therapeutic activity, the access of the investigator to patients, and many others factors which have to be taken into consideration when devising a research protocol. It is particularly useful at this meeting to be able to meet the person who is actually going to do the work since the initial approach may well have come from the 'dog's body's' head of department or consultant. If the research is likely to involve other members of staff from different departments such as biochemistry or haematology, these should be included at the beginning of protocol discussions rather than at the start of the trial when the realities of the additional work-load may suddenly dampen their enthusiasm. At an early stage of the discussion there must be a clear description of the objectives of the trial and hopefully these will be similar for the investigator and the company. Often, however, there has to be compromise because the company will invariably want to answer a basic question, but nevertheless one of major importance-does the drug work in condition $X$ ? Is it an efficacious treatment and is it safe? In other words, the company is asking questions which are vital to the drug's development and are regarded by many pharmaceutical physicians as the 'bread' of clinical research. The investigator, on the other hand, may want to answer questions relating to the drug's mode of action in vitro, which while of academic interest must be regarded as 'jam'. Of course the 'bread' research may on occasions lack originality and it is not unreasonable to improve the academic interest of the study by including some 'jam' but the balance has to be right for both parties.

The objectives of the trial form one of a number of elements which are essential to the clinical trial protocol and are listed in Table 2 . Investigators frequently demonstrate a remarkable naivety about the design of clinical trials and ignorance about methods of

Table 2 Essential elements of the clinical trial protocol

\begin{aligned} & \hline 1 Contents page \\ & 2 Background and general aims \\ & 3 Specific objective of trial \\ & 4 Patient Selection-inclusion criteria \\ &$\quad$-exclusion criteria \\ & 5 Trial design $\quad$ consent \\ & 6 Registration of patients and treatment allocation \\ & 7 Treatment schedules \\ & 8 Assessments \\ & 9 Administration and organization \\ & 10 Numbers of patients \\ & 12 Plans for statistical analysis \\ & 13 Case record form \\ & \hline\end{aligned}


statistical analysis and their interpretation. Even though excellent references (Pococks, 1983; Altman \& Gore, 1982) are available for enlightening those who feel that they have 3 such a gap in their knowledge, expert advice is always to be welcomed and most.

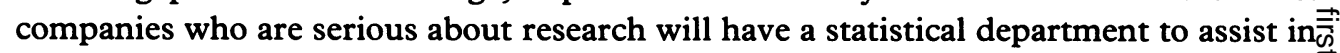
trial design and analysis.

A professional statistician should ideally be involved at an early stage in the design of the trial to help ensure that the protocol design and interpretation of the results conform to good scientific practice. All elements of the protocol are essential for the trial's $\varrho$ successful and efficient conduct. The statistician should be able to give useful advice on topics ranging from the design of record forms (Brigham, 1975; Wright \& Haybittle, $\overrightarrow{0}$ $1979 a, b, c$,$) to the calculation of sample sizes. The latter raises important ethical issues \overrightarrow{\vec{\omega}}$ since a study that is too large may expose an unnecessary number of patients to an ineffective treatment while small studies will be unable to detect clinically important $\mathrm{\Phi}^{\mathrm{B}}$. effects and therefore be of no scientific worth. Two types of error may result fromiv random variation and tolerable limits to the risks of these errors occurring should be $\omega$ defined in the protocol. A type 1 error occurs when it is concluded that there is a $\vec{\omega}$ difference between two treatments when, in fact, one does not exist. A probability of $0.05(p=0.05)$ represents a 1 in 20 chance of a type 1 error occurring and is the figure $\vec{c}$ usually quoted for statistical significance. A type 2 error occurs when it is concluded that no clinically important difference exists between treatments when in reality one exits. A® 1 in 10 chance of a type 2 error, known as a beta $(\beta)$ of $0 \cdot 1$, is usually consideredo acceptable. It is frequently defined as the power $(1-\beta)$ of the study, i.e. the probability of detecting a clinically important difference. Despite the importance of false negatives the beta value is rarely quoted in reports of clinical trials so that the unwary are easify misled. Thus in a review of 71 published randomized controlled trials which had failêto to detect a significant difference between groups, Freiman et al. (1978) found that 50 of them could have missed a $50 \%$ improvement.

In order to calculate sample size, it is necessary to define the power and the statistical ${ }_{\Omega}^{\frac{D}{\Phi}}$ significance level for the study and to have a clear opinion of the size of difference in $\vec{\circ}$ outcome between the experimental and control group which would be of clinical 3 significance. It is therefore apparent that a larger sample size will be necessary to detect a small difference than a large difference. Further factors which have to be taken into consideration when calculating sample size are:

- the variation inherent in the measurement of the variable of interest;

- the ratio of the numbers of patients on new $v s$ control treatment.

Actual numbers may be calculated using mathematical formulae (Donner, 1984; Lachin, 1981) but for the majority of us who are not mathematically minded nomograms have been published which are easy to use and provide a quick method for ascertaining sample size (Altman \& Gore, 1982; Young et al., 1983). Armed with this number and a reasonably clear definition of the entry criteria to be used for the trial it should be possible to estimate the likely duration of the trial if investigators make an educated guess as to the numbers of such patients passing through their hands. Such an $\mathrm{N}_{\mathrm{N}}$ estimate should be made cautiously since investigators are surprisingly inaccurate when predicting recruitment rates.

Over-optimistic targets which are later not met produce an unwanted sense of failure $\frac{\infty}{\mathbb{C}}$ and administrative problems, such as the rescheduling of financial support for the $\stackrel{\oplus}{+}$ 
study. For the same reasons, the logistics of the trial should be carefully worked out so that the investigator is not hopelessly over-burdened by, for example, having to make a large number of repeat observations on patients whose numbers are increasing rapidly because of successful recruitment. If this does occur then recruitment of patients may have to be halted temporarily with resultant delay. It is also useful to think about the implications of the study for ancillary staff, not only to see whether sufficient support is available from nursing and other services, but also so that the investigator may be forewarned about any training that may be necessary for such staff to play a part in the research.

At the end of these initial discussions it should be possible to start drafting a trial protocol. It is usual to expect the company to work on the drafts and to send copies to the investigator for comment. The protocol can be expected to be modified on many occasions before the final one is approved by all interested parties. Before the trial can begin, however, there are many preparations to complete.

\section{Preparing for the trial}

A patient in the UK cannot be given a drug in its research phase of development unless the clinical trial in which the patient will take part has been approved by the Department of Health and Social Security. Permission is granted (or refused) after consideration of the company's application for a clinical trial certificate (CTC) or clinical trial exemption (CTX). It is the responsibility of the company through its registration department to obtain this permission and it is clearly sensible to get an estimate from the company of the time that this procedure will take since it will affect directly the starting date of the trial.

The investigator will be expected to obtain ethical committee permission for the trial and it is unusual for ethical committees to consider protocols unless the CTC/CTX has been granted by the regulatory authority. Once approval has been given, the company and investigator will have to make sure that sufficient supplies of the drug have been ordered and that they can be correctly handled by the local pharmacy. The company will have been responsible for the ordering and correct packaging of supplies so that, for example, the blindness of patient and doctor are assured by providing identical packaging for placebo and active medications. After delivery, the investigator must be sure they will be correctly dispensed by giving the pharmacist a full briefing on the trial. When medication is being given double-blind, i.e. neither the investigator nor patient knows whether the medication is active or placebo, the company should supply a sealed envelope containing the treatment code to the investigator. The code is thus available if required in an emergency.

Before any patient is given the trial drug, the investigator should be satisfied that the company will take the responsibility for any injuries that may be caused to trial patients by the drug. The Association of the British Pharmaceutical Industry (ABPI, 1983) has published guidelines on compensation to patients involved in phase II and III trials and an investigator should request written confirmation that the company accepts these guidelines.

If the study is a complicated one, then it may often be useful to undertake a pilot 
study which will test the workability of the protocol by checking factors such aso dispensing procedures and the convenience of the forms used to record data on the study patients. It also presents a useful opportunity to see how easy it is to recruit? patients into the study and will thus assist in the estimation of the duration of the trial In addition, it may indicate that while the trial is perfectly feasible for the investigatorso it presents too much inconvenience for the subjects, e.g. the extra burden of repeated investigations may mean that the patient has to spend an unacceptable amount of time $\frac{0}{8}$ away from work. The 'dummy run' represents the last convenient chance to alter the protocol since this cannot be carried out, except for the most urgent of reasons, once the full scale trial has begun.

\section{The trial}

Given a well-thought-out protocol, a clear understanding of the logistics, good preparation before the trial and a little bit of luck, everything should go according toplan. The company will maintain a close interest in the progress of the trial and will bew keen to monitor patient flow, i.e. the numbers of patients entering and completing the trial, as well as aspects of data collection. In particular the company will be interested in $\vec{f}$ the completeness of data entry and the plausibility of the data which have beeno collected. For the company to achieve these objectives investigators should be easy to $\vec{P}$ contact and the same applies to the company monitor. An exchange of home phoneक्ष numbers is often a useful step in ensuring good communication. It is possible that in the future such monitoring will often be performed remotely from the investigators byoo using systems such as PRESTEL, where the investigator enters data directly into a cent computer to which the company monitor also has access (Waldron \& Cookson, 1984). The use of computers should not enable an individual patient to be identifiable from the data which have been collected. Patient anonymity should be maintained as with data collected conventionally.

Investigators should inform the company of changes in circumstances which may adversely affect the successful outcome of the trial. Such changes include closure of ${ }^{3}$ wards in a hospital, the movement of staff involved in the trial and unexpected expenditure. They also have a legal obligation to report immediately serious (fatal, life-threatening, disabling or incapacitating) adverse reactions in trial patients to the 3 licensing authority. If the product is being investigated under a CTX then minor: reactions are also required to be reported immediately but if the study is being conducted under a CTC, then such reactions need only be reported in summary at the end of the trial. The company also needs to know about adverse reactions since other trials may well be being conducted on the drug by other investigators who need to be informed. It is therefore usual in the first instance to inform the company straightaway; it will then take immediate steps to notify the regulatory authority on the investigators' behalf, although the investigators will still be expected to fill in adverse reaction reports personally.

\section{A successful outcome}

At the end of the trial, and preferably after each patient is completed, investigators 
should check the data that have been collected since if mistakes have been made, e.g. failure to record the age of the patient, it is only the investigators and not the company who can track the patient down to rectify the error. Measurements that are outside the normal range may be true or misrecorded but only investigators can pass judgement, so it is useful to have this opinion before the data are handed to the company. It is timeconsuming for the statistician to have to solicit an opinion at a later stage during analysis.

All investigators are eager for results but patience is required. Remember that a welldesigned and well-documented trial will be easier to analyse, so results come quicker to those who have planned well and documented the results carefully (and legibly). Even so there is a temptation to break the sealed code and start to make judgements based on only a small number of patients in the trial. However commendable such interest may be it should be avoided since hastily drawn conclusions cannot be substantiated until all the results are analysed.

When the analysis has been completed and the results interpreted clinically there is a tendency to file them away and forget them. Don't! Nothing is more irritating than waiting weeks or months for an investigator to write up trial results, the wait often being interspersed with telephonic pledges of manuscripts 'in the post', 'on final draft' or 'ready for typing when the department's secretary returns from sick leave'.

If there are problems in writing up the study, consult the company who should be prepared to assist with secretarial support, reference searches, etc. It is after all in the company's interest to give as much assistance as possible to the investigator.

This article has been written with the intention of encouraging the reader who is interested in research to consider collaboration with the pharmaceutical industry. The industry is committed to supporting medical research and is always eager to listen to new ideas.

\section{ACKNOWLEDGEMENTS}

I am grateful to Dr R. Cookson, Dr M. Emanuel and Mrs A. Whitehead for their helpful comments on the manuscript and to Miss T. Sloan for typing it.

\section{REFERENCES}

Altman D. G. \& Gore S. M. (1982) Statistics in Practice. London, British Medical Association.

Association of the British Pharmaceutical Industry (1983) Compensation and drug trials. British Medical fournal 287, 675.

Brigham F. R. (1975) Some quantitative considerations in questionnaire design and analysis. Applied Ergonomics 6, 90-6.

Donner A. (1984) Approaches to sample size estimation in the design of clinical trials-a review. Statistics in Medicine 3, 199-214.

Freiman J. A., Chalmers T. C., Smith H. \& Kuebler R. R. (1978) The importance of beta, the type II error and sample size in the design and interpretation of the randomized control trial: survey of 'negative' trials. New England fournal of Medicine 299, 690-4. 
Lachin J. M. (1981) Introduction to sample size determination and power analysis for clincial trials $\frac{\mathbb{Q}}{\mathbb{Q}}$ Controlled Clinical Trials 2, 93-113.

Pocock S. J. (1983) Clinical Trials. Chichester, John Wiley \& Sons.

Young M. J., Bresnitz E. A. \& Strom B. L. (1983) Sample size nomograms for interpreting negative clinical $\overrightarrow{\vec{B}}$
studies. Annals of Internal Medicine 99, 248-51.

Waldron H. A. \& Cookson R. F. (1984) Use of a viewdata system to collect data from a multicentre clinicalo trial in anaesthesia. British Medical fournal 289, 1059-61.

Wright P. \& Haybittle J. (1979a) Design of forms for clinical trials (1). British Medical fournal 2, 529-30.

Wright P. \& Haybittle J. (1979b) Design of forms for clinical trials (2). British Medical fournal 2, 590-2.

Wright P. \& Haybittle J. (1979c) Design of forms for clinical trials (3). British Medical fournal 2, 650-1.

Received 1 March 1985; accepted for publication 29 March 1985 